\title{
A novel formulation of an approximate valence bond model (AVB2) and its application to the tautomeric forms of porphyrin and porphycene
}

\author{
Marta Hallay-Suszek ${ }^{1}$ (D) . Paweł Grochowski ${ }^{1}$ - Bogdan Lesyng ${ }^{2,3}$
}

Received: 4 December 2018 / Accepted: 29 March 2019 / Published online: 10 May 2019

(C) The Author(s) 2019

\begin{abstract}
This study presents a novel formulation of the approximate valence bond method, which can be applied as a very fast generator of the molecular potential energy function. The AVB2 model was formulated and parameterized for porphyrin and porphycene using results of quantum mechanical computations at the B3LYP/6-31G $(\mathrm{d}, \mathrm{p})$ level. The DFT potential energy, its gradients, and the Hessian-matrix elements, as well as effective atomic charges at local energy minima and transition states, were used for the parameterization of the AVB2 Hamiltonian matrix. The AVB2 method, and in particular its anharmonic version, very well reproduce the potential energy maps for all representative geometries of the studied systems, including harmonic frequencies, and possible proton translocations. For validation of the method, we performed molecular dynamics simulations for isolated molecules accounting for internal double proton transfer processes, which are strongly correlated with changes of the electronic charge density. The simulated power spectra were compared with the experimental infrared spectra. More precise simulations of IR spectra at the classical and quantum dynamics levels, as well as extensions of the AVB2 parameterization to electronic excited states, are the subject of further research.
\end{abstract}

Keywords AVB2 - Quantum computations · Potential energy surface parameterization · Proton transfer ·

Molecular dynamics · Porphycene · Porphyrin

\section{Introduction}

Most quantum-mechanical studies of larger (bio)molecular and nanosystems are carried out based on the Born-Oppenheimer approximation. This allows, in particular, to determine the

This paper belongs to Topical Collection 8th conference on Modeling \& Design of Molecular Materials (MDMM 2018)

Electronic supplementary material The online version of this article (https://doi.org/10.1007/s00894-019-4022-7) contains supplementary material, which is available to authorized users.

Marta Hallay-Suszek

hallay@icm.edu.pl

1 Interdisciplinary Centre for Mathematical and Computational Modelling, University of Warsaw, Pawińskiego 5a, 02-106 Warsaw, Poland

2 Department of Biophysics, Institute of Experimental Physics, Faculty of Physics, University of Warsaw, Pasteura 5, 02-093 Warsaw, Poland

3 Bioinformatics Laboratory, Mossakowski Medical Research Centre, Polish Academy of Sciences, Pawińskiego 5, 02-106 Warsaw, Poland quantum potential energy surface (Q-PES) for ground and/or excited states. Q-PES depends on the atomic positions (molecular geometry), that can be further applied to classical molecular dynamics simulations (MD) or to Monte Carlo (MC) sampling, to obtain time-averaged or ensembleaveraged molecular properties. We will be using the name Q-PES in order to clearly indicate its quantum origin, unlike PES, which is usually based on semi-empirical forcefield parameters, widely used in macromolecular MD or MC simulations. Q-PES, unlike PES, is not limited to a fixed topology of a molecule, it can describe the breaking of chemical bonds or the creation of new ones. Simulations of chemical reactions, including proton transfer processes, require high-level quantum mechanical calculations that are time-consuming. A typical methodological approach to such studies can be divided into two steps. The first step refers to high-level QM calculations to determine the local QPES shape for the selected stationary states. The second one refers to simulations of classical or quantum atomic (nuclear) motions. For simplicity, these approaches will be referred to as MD/Q-PES or QD/Q-PES. Note that analytical approximations of Q-PES can also be used in conventional $\mathrm{MM}$ and MD studies, and this is one of possible practical 
solutions for time-consuming simulations. Such approximation for smaller molecular systems can, for example, be obtained from the Shepard's interpolation of the local Taylor Q-PES expansion up to the second-order Hessian, or to higher terms [12]. The method was developed for local internal coordinates [15], as well as for Cartesian coordinates [14]. In the latter case, PES symmetry with respect to molecular rotation has been ensured by the invariant integral method, but it substantially increases the computational cost of analytical potential derivatives. One can also design a multivariate PES approximation using other interpolation methods, see e.g., [10] and references therein. It should be noted, however, that such approaches have problems with the ability to transfer parameters from smaller molecular fragments to larger systems. For this reason, conventional databases of molecular "force-fields" typically do not contain such parameterizations. As for other practical approaches the Car-Parrinello molecular dynamics (CPMD) method should be indicated (see e.g., [11] for an overview) which, in particular, has been used to model molecular systems with intramolecular proton transfer, e.g., to porphycene and to its isotopomers [17, 20, 21]. CPMD belongs to the MD/Q-PES class, although this hybrid variational approach does not require diagonalization of the Hamiltonian matrix at each MD time-step, and is therefore faster than other conventional MD/Q-PES approaches, in particular faster than ab intio Born-Oppenheimer MD. It allows also for quantum delocalization of nuclei with the use of path-integral formalism (PIMD). However, CPMD and in particular PIMD, are not fast enough to be used for larger (bio)molecular systems and long MD or QD simulations. Therefore, when thinking of quantum-based physics of a PES and its applications for MD or QD simulations, attention should be paid to the very fast approximate valence bond (AVB) method, which was previously developed and applied for enzymatic reactions with proton transfer [2, 9, 16] and with some improvements [8]. Instead of parameterizing a Born-Oppenheimer PES to get its analytical approximation, it is much more effective to develop and apply an analytical approximation of the electronic Hamiltonian matrix, using a low-dimensional functional base, e.g., applying a valence-bond formalism. The original idea of such approach comes from A. Warshel and coworkers, see e.g., [13, 18, 19], who formulated the empirical valence bond method (EVB). It should be noted, however, that the parameterization of Hamiltonian matrix elements using empirical parameters, which are based on ensemble averaged properties, raises some doubts. It is much safer to design a fast quantum generator of the potential energy function that is able to mimic high-level QM calculations, and take into account the influence of a molecular environment using molecular fields perturbing the quantum-domain Hamiltonian. This study further develops the AVB method for proton transfer processes [2, 9, 16] and applies it to two model systems: porphyrin and porphycene with an intramolecular double proton transfer. The aim is to accurately reproduce high-level ab initio calculations using an approximate low-dimensional Hamiltonian matrix, in particular the use of parameterization of Hessian matrix elements in stationary geometries. The method can be implemented using any coordinates, and in this study we apply a combination of internal and Cartesian coordinates. We use normal transformation [7] to ensure rotational invariance of Q-PES, without increasing the cost of analytical derivatives, for any number of atoms. The novel approach will be referred to as AVB2. The AVB2-PESes can further be used in MD, QD or QCMD simulations to reproduce and/or predict a wide range of molecular properties of these model molecules, including spectroscopic ones. Very preliminary applications of MD/AVB2PES in the prediction of IR spectra are presented in this study. The next research will focus on more advanced simulations, including QCMD ones. It should also be emphasized that the methodology used to design these prototype AVB2PESes, can be further applied to other molecules or molecular fragments, as well as to the design of Q-PESes of much larger molecules consisted of molecular fragments, already described by the known Q-PESes.

\section{Model systems and DFT calculations}

Our model systems are the porphyrin and porphycene molecules in their ground electronic state in vacuum. Since we have already been involved in studies of these molecules [5, 17], this study is a natural extension of our previous analyzes. It should also be emphasized that due to the delocalization of protons in the molecular cavities of these molecules, which results in large changes in electron densities, these model molecules are difficult prototype-cases to design effective approximate AVB Hamiltonians. The Gaussian 03 program [4] was applied for DFT optimization of molecular geometries, as well as for calculations of Hessians. The spin-restricted B3LYP functional [3] and 6-31G(d,p) basis set were used. Optimized geometries are presented in Figs. 1(porphyrin), 2 and 3(porphycene). Geometry types, their symmetries, and relative energies are listed in Table 1. In Table 1, we also provide the number of distinct stationary points on Q-PESes, which represent geometry of the same type, but differ in the locations of individual protons with respect to the nitrogen atoms. For example, porphyrin has four stable trans geometries (Mt), which are starting points of 16 reaction paths (single proton transfers) passing through the saddle points (S1), and ending in eight stable cis points (Mc). In addition, it has eight reaction paths (double proton transfers) passing through four saddle points 


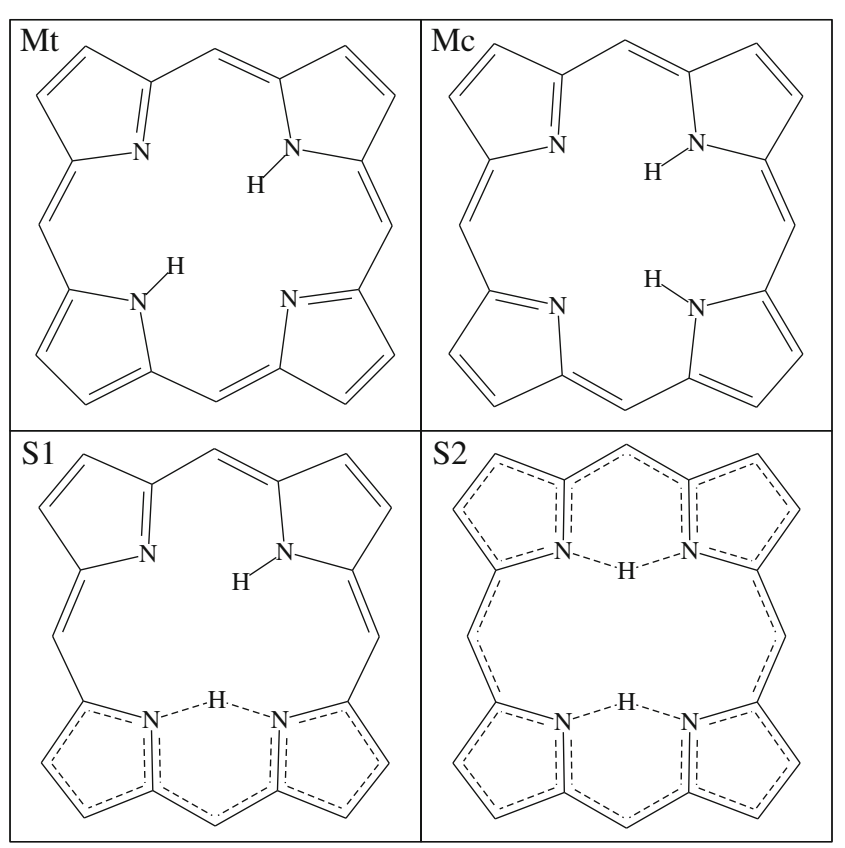

Fig. 1 Stationary structures of porphyrin. For denotations see Table 1. Stationary structures of porphyrin. In the upper row, the stationary states of porphyrin correspond to two minima: the trans and the cis configurations, Mt and Mc respectively; in the lower row, the saddle points of the 1st and 2nd orders, S1 and S2 respectively. The transition state $\mathrm{S} 1$ connects the trans and cis minima, while the transition state $\mathrm{S} 2$ connects two minima of the trans types, as well as two minima of the cis types

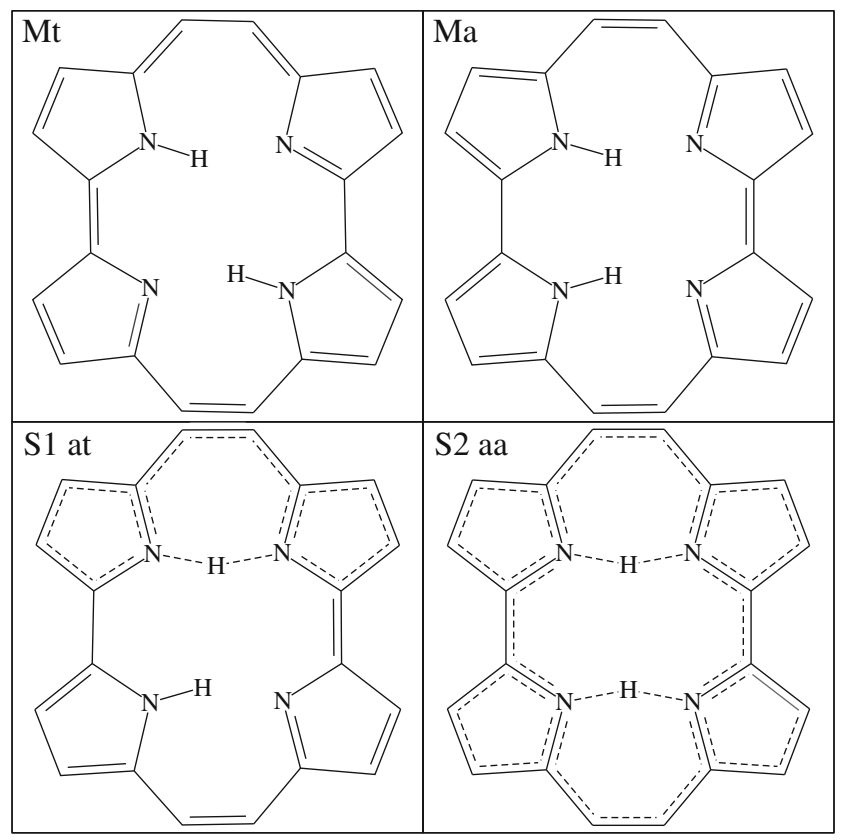

Fig. 2 Low-energy stationary structures of porphycene. In the upper row, the stationary states of porphycene correspond to two minima: the trans and the cis- $a$ configurations, Mt and Ma, respectively; in the lower row - the saddle points of the 1st and 2nd orders, S1-at and S2$\mathrm{aa}$, respectively. The transition state $\mathrm{S} 1$-at connects the trans and cis-a minima while the transition state $\mathrm{S} 2$-aa connects two minima of the cis-a types

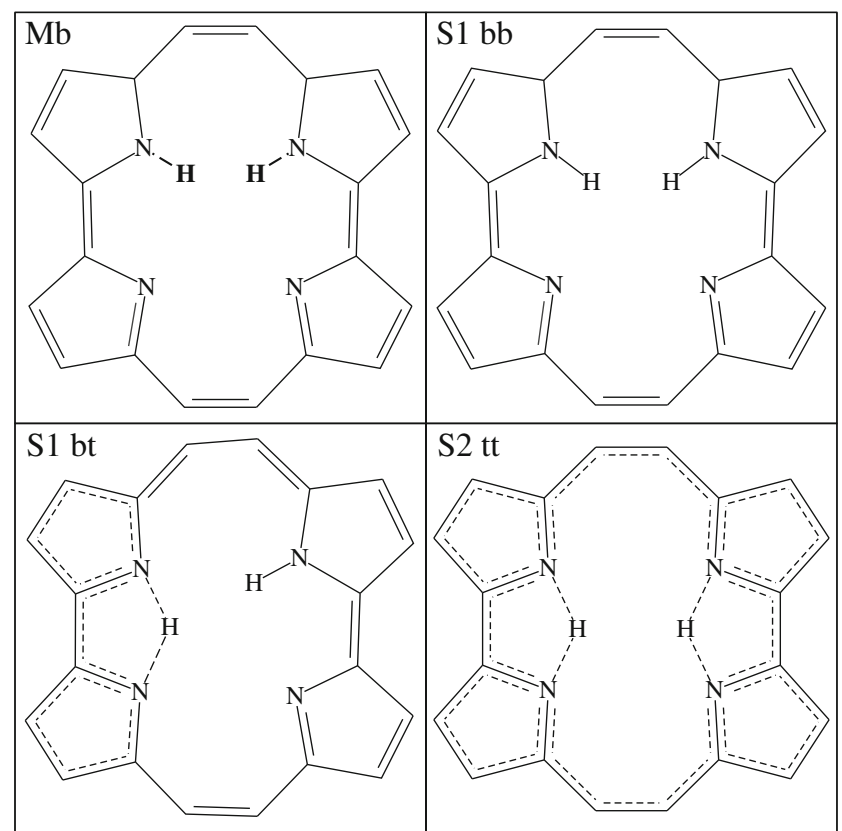

Fig. 3 High-energy stationary structures of porphycene. In the upper row, the stationary states of porphycene corresponds to the $c i s-b$ minimum (it should be noted that it is the only structure that is not planar) and the saddle point of the 1st order, $\mathrm{Mb}$ and S1-bb respectively; in the lower row, the saddle points of the 1st and 2nd orders, S1-bt and S2-tt, respectively. These stationary states are not taking to the parameterization process because in room temperature these are not accessible

(S2) and interconnecting a pair of Mt points, as well as a pair of Mc points. Porphycene is more complex because of two distinct stable cis forms (Ma and $\mathrm{Mb}$ ). Some of these geometries are accessible at room temperature, when the total energy is of the order of $3 N_{A} k T \simeq 283 \mathrm{~kJ} / \mathrm{mol}\left(N_{A}\right.$ $=38$ is the total number of atoms). We are interested in the processes occurring in the low temperatures, below the total energy level of $100 \mathrm{~kJ} / \mathrm{mol}$. Porphycene has 18 stationary points in this range, but they fall into two separate domains of Q-PES, without the interconnecting paths. We further consider the dynamics in one domain with nine points only (2Mt, 2Ma, 4S1ta, S2aa), which excludes the exchange of protons in porphycene.

We also performed B3LYP/6-31G(d,p) DFT calculations of the two-dimensional Q-PES cross section for porphyrin. The molecule was fixed in the Mt geometry, with the exception of one cavity proton, which is moved on the twodimensional regular grid in the plane of the molecule. The resulting protonic map is presented in Fig. 4.

\section{The AVB2 potential energy}

Consider a molecular system consisting of $N_{A}$ atoms with an approximate analytical Q-PES $E(\mathbf{r})$, where $\mathbf{r}$ 
Table 1 Stationary geometries of porphyrin and porphycene, and the number of stationary points on the Q-PES

\begin{tabular}{|c|c|c|c|c|c|}
\hline Molecule & Geometry & $E[\mathrm{~kJ} / \mathrm{mol}]$ & Symmetry & No. of points & LHA parameters \\
\hline \multirow[t]{4}{*}{ Porphyrin } & $\mathrm{M} \mathrm{t}$ & 0.0 & $\mathrm{D}_{2 h}$ & 4 & $1+19+913$ \\
\hline & $\mathrm{Mc}$ & 34.3 & $\mathrm{C}_{2 v}$ & 8 & $1+37+1769$ \\
\hline & $\mathrm{S} 1 \mathrm{tc}$ & 63.9 & $\mathrm{C}_{s}$ & 16 & $1+74$ \\
\hline & $\mathrm{S} 2 \mathrm{tt}, \mathrm{cc}$ & 94.2 & $\mathrm{D}_{2 h}$ & 4 & $1+19$ \\
\hline \multirow[t]{8}{*}{ Porphycene } & $\mathrm{M} \mathrm{t}$ & 0.0 & $\mathrm{C}_{i}$ & 4 & $1+38+1768$ \\
\hline & $\mathrm{M} \mathrm{a}$ & 9.3 & $\mathrm{C}_{2 v}$ & 4 & $1+37+1767$ \\
\hline & $\mathrm{S} 1 \mathrm{ta}$ & 17.2 & $\mathrm{C}_{s}$ & 8 & $1+74$ \\
\hline & S2 aa & 25.7 & $\mathrm{D}_{2 h}$ & 2 & $1+19$ \\
\hline & $\mathrm{Mb}$ & 126.3 & $\mathrm{C}_{2}$ & 8 & $1+56+3136$ \\
\hline & $\mathrm{S} 1 \mathrm{bb}$ & 138.4 & $\mathrm{C}_{2 v}$ & 4 & $1+37$ \\
\hline & $\mathrm{S} 1 \mathrm{bt}$ & 183.4 & $\mathrm{C}_{s}$ & 8 & $1+74$ \\
\hline & $\mathrm{S} 2 \mathrm{tt}$ & 269.3 & $\mathrm{D}_{2 h}$ & 2 & $1+19$ \\
\hline
\end{tabular}

The geometry types are: M - minimum, S1(2) - saddle point of the 1st (2nd) order, $\mathrm{t}$ - trans, $\mathrm{c}$ - cis form, a, $\mathrm{b}$ - two distinct cis forms in porphycene

is $3 N_{A}$-dimensional vector of Cartesian coordinates. We assume that the system is isolated, and Q-PES should be invariant with respect to system's translations and rotations. We construct the invariant function in the form $E(\hat{R} \mathbf{r}+\mathbf{v})$, where the matrix $\hat{R}$ and vector $\mathbf{v}$ represent rotation and translation, respectively, of the whole system to the so-called normal orientation. The normal orientation minimizes the geometrical deviation with respect to the fixed reference geometry $\mathbf{r}_{0}$,

$\left|\hat{R} \mathbf{r}+\mathbf{v}-\mathbf{r}_{o}\right|^{2}=\min$.

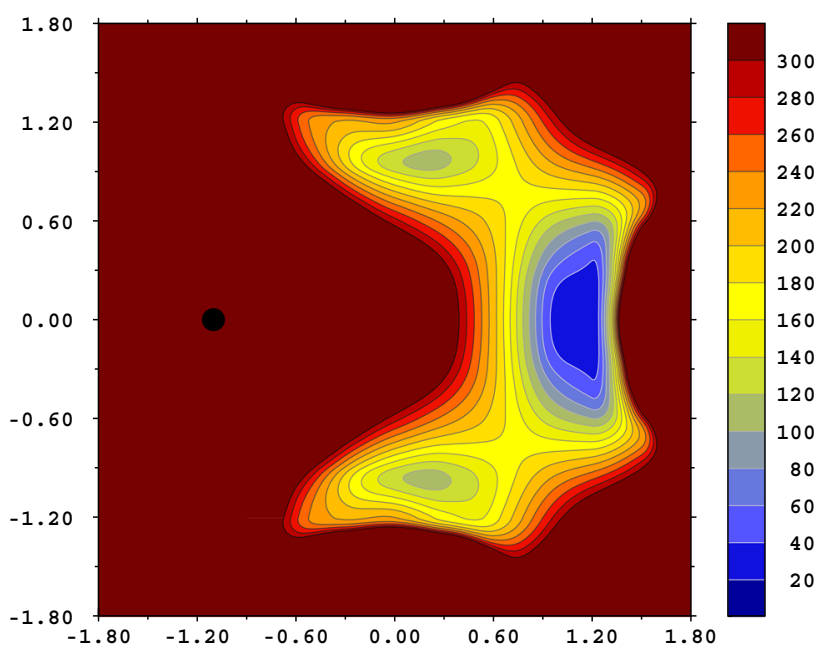

Fig. 4 Protonic potential energy map (DFT-PES) for porphyrin in Mt geometry. DFT-PES was obtained by moving the right inner hydrogen in the plane of the molecule $(x, y)$ in the cavity region: $x \in(-1.8 ; 1.8), y \in(-1.8 ; 1.8)$, while all other atoms were fixed in space. The coordinates of nitrogens are $(0, \pm 2.07)$ and $( \pm 2.07,0)$. The second proton is fixed at $(-1.06,0)$. Mt geometry corresponds to position $(1.06,0)$ for the moving proton. The coordinates are in $\AA$, the energy levels are in $\mathrm{kJ} / \mathrm{mol}$. Potential energies over $300 \mathrm{~kJ} / \mathrm{mol}$ are plotted in dark red
The analytical expressions for $\hat{R}$ and $\mathbf{v}$ are known [14], as well as their analytical first and second derivatives with respect to $\mathbf{r}$ [7]. The choice of $\mathbf{r}_{0}$ is arbitrary, but for convenience we use the stationary geometry with the highest point symmetry. The standard orientation implemented in some quantum-mechanical programs is not useful here because in some cases (degenerated tensor of inertia) the corresponding geometry transformation is not continuous and not differentiable.

Suppose the system has $N_{M}$ stable geometries, which represent the local minima of Q-PES, and are denoted $\mathbf{r}_{i i}$ $\left(i=1, \ldots, N_{M}\right)$. It is assumed hereafter that all stationary geometries, including the stable and saddle points, are provided in the normal orientation. We define $E(\mathbf{r})$ as the lowest eigenvalue of a symmetric real-valued square electronic Hamiltonian matrix $\hat{H}(\mathbf{r})$ of the order $N_{M}(i=$ $\left.1, \ldots, N_{M}\right)$,

$\sum_{j=1}^{N_{M}}\left[H_{i j}-\delta_{i j} E\right] c_{j}=0$,

where $\mathbf{c}$ is the corresponding normalized eigenvector. The idea of parameterizing the potential through the matrix $\hat{H}$ is illustrated in Fig. 5. The element $H_{i i}(\mathbf{r})$ represents a fixed electronic valence state and it is responsible for the local minimum of Q-PES at $\mathbf{r}_{i i}$, in the sense that for $\mathbf{r} \simeq \mathbf{r}_{i}$. The local minima are parameterized up to the second order in Taylor expansion,

$H_{i i}=\frac{1}{2} \mathbf{x}_{i}^{T} \hat{\mathbf{A}}_{i} \mathbf{x}_{i}+\mathbf{B}_{i i}^{T} \mathbf{x}_{i}+C_{i i}+V_{i}$,

where $\mathbf{x}_{i}$ is the $3 N_{A}$-dimensional vector of generalized atomic coordinates, specific for the $i$ th valence state, $\hat{\mathbf{A}}$, 


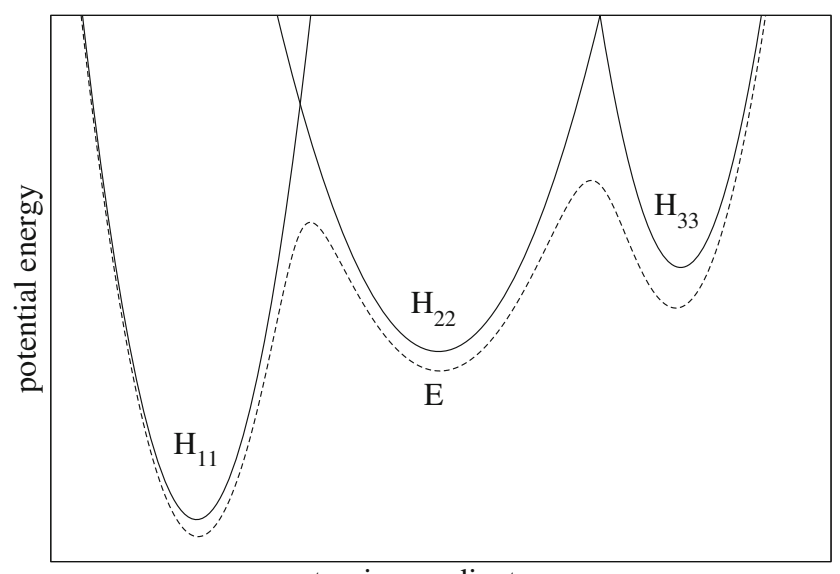

atomic coordinates

Fig. 5 Schematic construction of the AVB-PES potential energy (dashed line), as the ground-state eigenvalue of the matrix $H$, of the order 3. The local minima are related to the diagonal elements of the $H$ matrix. The shapes of the energy barriers are controlled by the off-diagonal elements $H_{12}$ and $H_{23}$

B and $C$ are the parameters of the matrix, vector and scalar type, respectively, and $V_{i}$ is an additional potential energy term which will be described later. Hereafter we consider two versions of the model. In the first one, called the local harmonic approximation (LHA), the generalized coordinates are equal to the relative Cartesian coordinates, $\mathbf{x}_{i}=\mathbf{r}-\mathbf{r}_{i i}$. In the second version, called the non-harmonic approximation (NHA), we also apply the relative Cartesian coordinates for all atoms, except for the mobile protons. The proton's coordinates in NHA are,

$x_{1}=1-\exp \left[a\left(d_{i}-d\right)\right]$

$x_{2}=\alpha \exp \left[b\left(d_{i}^{2}-d^{2}\right)\right]$,

$x_{3}=\beta \exp \left[b\left(d_{i}^{2}-d^{2}\right)\right]$,

where $a$ and $b$ are parameters, $d$ and $d_{i}$ denote distance between the proton and its binding nitrogen atom $(\mathrm{NH}$ distance) in geometries $\mathbf{r}$ and $\mathbf{r}_{i i}$, respectively, whereas $\alpha$ and $\beta$ are angles which describe deviation of the $\mathrm{NH}$ vector in $\mathbf{r}$ from the direction of the same vector in $\mathbf{r}_{i i}$. The angle $\alpha$ is measured in the plane defined by the nitrogen and its two neighbor carbon atoms (CNC plane), and the angle $\beta$ is measured in the plane perpendicular to the $\mathrm{CNC}$ plane. This choice of the protonic generalized coordinates in NHA ensures the Morse-type potential for the protonacceptor bond and curved shape of the protonic potential well, which is more realistic than the shape resulting from LHA, compare Figs. 4 and 6.

In the LHA model $V_{i}$ is equal to the quartic angular potential $V_{q a}$, which was designed to strength stabilization of the co-planar geometry of pyrole rings and protons. It has quartic form, which does not influence the Hessian,

$$
\begin{aligned}
V_{q a} & =B_{1} \sum_{k} \sin ^{4} \theta_{k}+B_{2} \sum_{l} \sin ^{4} \phi_{l} \\
& +B_{3} \sum_{k, l}\left(\sin ^{3} \phi_{k} \sin \phi_{l}+\sin \phi_{k} \sin ^{3} \phi_{l}\right),
\end{aligned}
$$

where $\theta$ and $\phi$ are dihedral angles, which are equal to zero in the planar stationary geometries, and $B_{1,2,3}$ denotes the parameters. The $\theta$ angles are defined by the following atoms: $\mathrm{NCC}_{x} \mathrm{C}$ in porphyrin (8 angles), or NCCC (4), $\mathrm{NCC}_{x} \mathrm{C}_{x}$ (4), $\mathrm{CC}_{x} \mathrm{C}_{x} \mathrm{C}(2), \mathrm{CCCC}(2)$ and $\mathrm{C}_{x} \mathrm{C}_{x} \mathrm{CC}$ (4), CNCC (4), $\mathrm{CNCC}_{x}$ (4), NCCN (2) in porphycene (26 angles), where $\mathrm{C}_{x}$ denote atoms, which interconnect the pyrrole subunits. The $\phi$ angles are improper dihedrals defined by the HNCC atoms, and describe deviation of the protons from the CNC planes. The index $l$ runs through eight angles defined by each proton and each CNC group, so that as the potential $V_{q a}$ is independent on the valence state, and it is additive component of $E(\mathbf{r})$.

In the NHA model $V_{i}$ is sum of several terms, including: $V_{q a}$, the non-bonding potentials $V_{n b}$ for proton-heavy atoms interactions, and the proton-proton interaction potential $V_{p p}$. The non-bonding interactions are parametrized with the Morse function,

$V_{n b}(d)=D\left[e^{2 c\left(d_{o}-d\right)}-2 e^{c\left(d_{o}-d\right)}\right]$,

where $d$ is the interatomic distance, and $D, b$ and $d_{o}$ are the parameters, which depend on the type of heavy atoms. The list of non-bonding interactions depends on the valence state: the potential is applied between the proton and all heavy atoms, except the three atoms from the $\mathrm{CNC}$ group which binds the proton in the given valence state. The proton-proton interaction potential is,

$V_{p p}(d)=\frac{k e^{2}}{d} e^{-c^{\prime} d}$,

where $k$ is the Coulomb constant, $e$ is the elementary charge, $c^{\prime}$ is the parameter, and $d$ is the interprotonic distance.

Due to the linear term and the non-bonding potentials in Eq. 3, the minimum of $H_{i i}$ is usually not exactly in $\mathbf{r}_{i i}$, but this displacement is compensated by the coupling with other valence states, so that the resulting Q-PES ( $E=$ $\mathbf{c}^{T} \hat{H} \mathbf{c}$, where $\left.c_{i}\left(\mathbf{r}_{i i}\right)<1\right)$, has the local minimum at $\mathbf{r}_{i i}$. The coupling is caused by the non-diagonal Hamiltonian elements, which are used to shape the Q-PES between the local minima, and are parameterized applying the following form,

$H_{i j}=\exp \left(-\sigma\left|\mathbf{x}_{p}\right|^{2}\right)\left(\mathbf{B}_{i j}^{T} \mathbf{x}_{i j}+C_{i j}\right)$,

where $\sigma, \mathbf{B}$, and $C$ are the parameters; $\mathbf{x}_{i j}=\mathbf{r}-\mathbf{r}_{i j}, \mathbf{r}_{i j}$ denotes the transition geometry (the saddle point) between $\mathbf{r}_{i i}$ and $\mathbf{r}_{j j}, \mathbf{x}_{p}$ denotes the subset of $\mathbf{x}_{i j}$ with the six protonic 
Fig. 6 Protonic potential energy, AVB2-PES, for porphyrin in Mt geometry. For details see caption to Fig. 4. Upper and lower plot refer to the LHA and NHA models, respectively
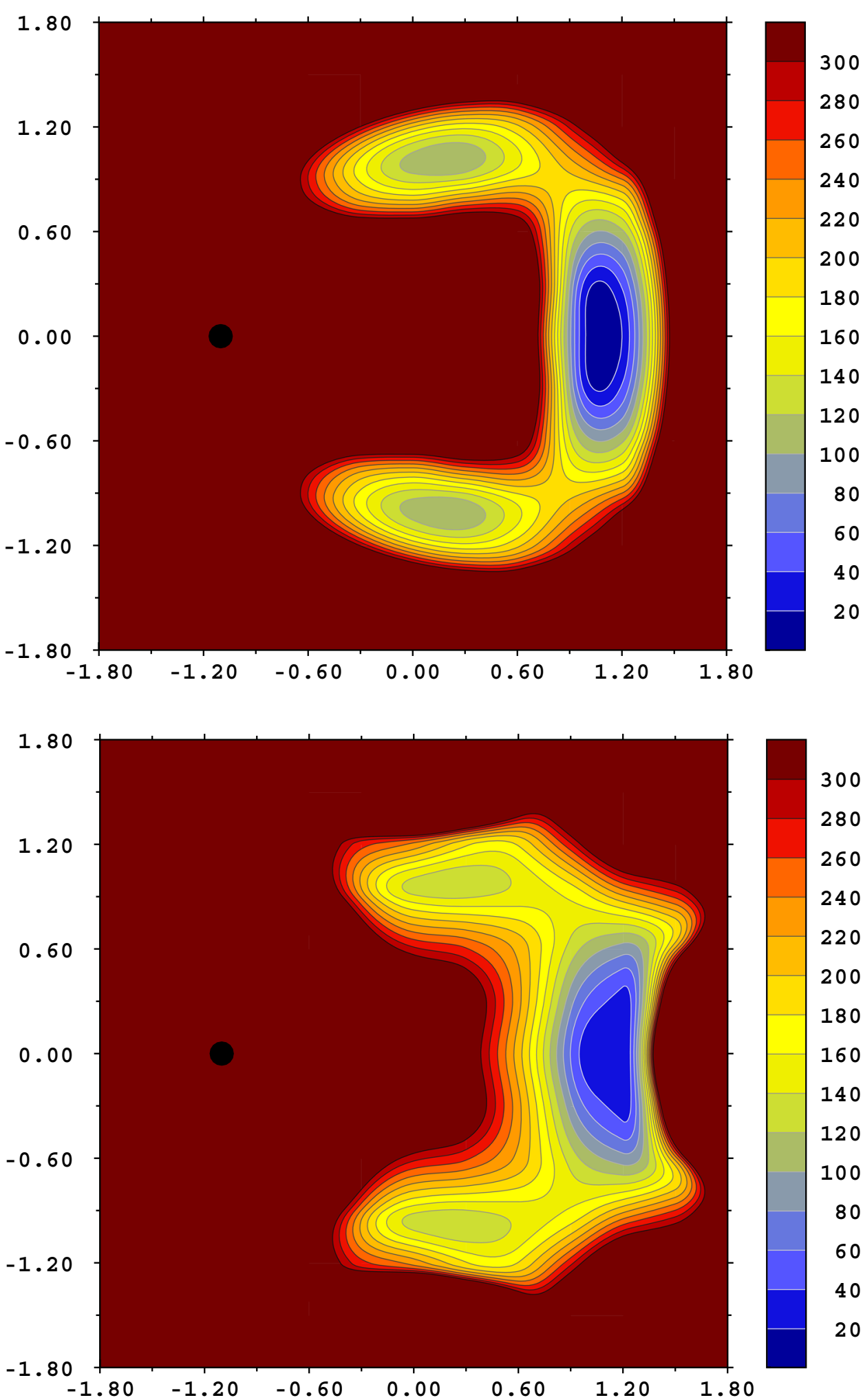

coordinates. In the LHA, we set $\sigma=0$. The off-diagonal elements of $\hat{H}$ are parameterized only for those pairs of Q-PES minima which are separated by a single energy barrier. All other off-diagonal elements are set to zero. The shape of $E(\mathbf{r})$ in a neighborhood of a saddle point $\mathbf{r}_{i j}$ is mainly determined by the elements $H_{i i}, H_{j j}, H_{i j}$, which corresponds to $c_{i}(\mathbf{r}) \simeq c_{j}(\mathbf{r}) \simeq 0.5$ and $c_{k \neq i, j}(\mathbf{r}) \simeq 0$ for $\mathbf{r} \simeq \mathbf{r}_{i j}$ 
The analytical gradient and Hessian of Q-PES can be computed based on the perturbation calculus,

$\mathbf{E}^{\prime}=\frac{\partial H_{i j}}{\partial \mathbf{r}} c_{i} c_{j}$,

$\hat{\mathbf{E}}^{\prime \prime}=\sum_{i, j=1}^{N_{M}} \frac{\partial^{2} H_{i j}}{\partial \mathbf{r} \partial \mathbf{r}^{T}} c_{i} c_{j}+\sum_{n=2}^{N_{M}} \frac{2 \mathbf{F}_{n} \mathbf{F}_{n}^{T}}{E-E^{(n)}}$,

where $E^{(n)}\left(n=2, \ldots, N_{M}\right)$ are higher eigenvalues of $\hat{H}$, $\mathbf{F}_{n}$ is an auxiliary denotation,

$\mathbf{F}_{n}=\sum_{i, j=1}^{N_{M}} \frac{\partial H_{i j}}{\partial \mathbf{r}} c_{i} c_{j}^{(n)}$,

and $c^{(n)}$ are eigenvectors of $\hat{H}$ corresponding to the higher eigenvalues. The derivatives of $E(\hat{R} \mathbf{r}+\mathbf{v})$, are computed from derivatives of $E(\mathbf{r})$ with help of the normal transformation formulae [7].

The AVB method describes also the atomic charges, and interaction of a molecule with an external electric field. The atomic charges are calculated from the following expression $\left(a=1, \ldots, N_{A}\right)$,

$q_{a}(\mathbf{r})=\sum_{i=1}^{N_{M}} c_{i}(\mathbf{r})^{2} q_{a i}$,

where $q_{a i}$ are parameters, which can be selected so that $q_{a}\left(\mathbf{r}_{i i}\right)$ are equal to the atomic charges derived from the quantum mechanical calculations, e.g., the ESP charges. The interaction of the molecule with the external electrostatic field can be described either by including the following additional terms in the AVB Hamiltonian

$\Delta H_{i i}=\sum_{a=1}^{N_{A}} q_{a i} V_{a}$,

or by adding the following approximate term to the total AVB energy

$\Delta E=\sum_{a=1}^{N_{A}} q_{a}(\mathbf{r}) V_{a}$,

where $V_{a}$ denotes the potential of the external electric field in location of atom $a$. In the first case, the AVB atomic charges change due to the external field, which corresponds to the molecular electronic polarization. In the second case, we account only for the static interaction, and ignore energy terms of the second-order with respect to the external electric field.

\section{Optimization of the parameters}

The basic LHA model includes the $C_{i j}, \mathbf{B}_{i j}$ and $\hat{\mathbf{A}}_{i i}$ parameters, where $i, j=1, \ldots, N_{M}$. The parameters are optimized by fitting the AVB potential values, gradients and Hessians to the reference B3LYP/6-31G(d,p) DFT data. The optimization is based on the minimization of the following quantities,

$\Upsilon_{1}^{2}=\frac{1}{N_{T}} \sum_{i j}\left[E\left(\mathbf{r}_{i j}\right)-E_{i j}\right]^{2}$,

$\Upsilon_{2}^{2}=\frac{1}{N_{T}} \sum_{i j}\left|\mathbf{E}^{\prime}\left(\mathbf{r}_{i j}\right)\right|^{2}$

$\Upsilon_{3}^{2}=\frac{1}{N_{M}} \sum_{i}\left\|\hat{\mathbf{E}}^{\prime \prime}\left(\mathbf{r}_{i i}\right)-\hat{\mathbf{T}}_{i i}\right\|_{(k)}^{2}$,

where $N_{T}$ denotes the total number of stationary points, $E_{i j}$ and $\hat{\mathbf{T}}_{i j}$ denote the DFT energies and Hessians, respectively, in the stationary points, and $k=0,1,2$ is a parameter that controls the fitting, see details further below. In the case of porphyrin, we have $N_{M}=12$ and $N_{T}=32$, in the case of porphycene low temperature regime $N_{M}=4$ and $N_{T}=9$. In each point in principle we have $1+3 N_{A}+$ $N_{A}\left(N_{A}+1\right) / 2=6670$ LHA parameters. This number can be significantly reduced taking into account molecular symmetries of the stationary geometries (see Table 1). We use the following expansions,

$\mathbf{B}_{i j}=\sum_{n}^{\prime} B_{i j n} \mathbf{V}_{i j n}$

$\hat{\mathbf{A}}_{i i}=\sum_{n \leq k}^{\prime} A_{i i n k}\left(\mathbf{V}_{i i n} \mathbf{V}_{i i k}^{T}+\mathbf{V}_{i i k} \mathbf{V}_{i i n}^{T}\right)$,

where $\mathbf{V}_{i j n}\left(n=1, \ldots, 3 N_{A}\right)$ denotes $3 N_{A}$-dimensional eigenvectors of $\hat{\mathbf{T}}_{i j}, A$ and $B$ denote independent scalar parameters, and the primes indicate that the terms which violate the point symmetry of Q-PES are excluded from the sums. The above expansions allow to describe the stationary points of the same type with the same set of parameters. The total number of LHA parameters ( $C$ plus $B$ plus $A$ ), which describe particular stationary points of porphyrin and porphycene are provided in Table 1. In Eq. 17 we use the denotation of a special norm,

$\|\cdot\|_{(k)}^{2}=\sum_{n=1}^{3 N_{A}} \frac{1}{\left|\tilde{w}_{i i n}\right|^{k}}\left|(\cdot) \mathbf{V}_{i i}^{(a)}\right|^{2}$,

where $w_{i i n}$ denotes the eigenvalue corresponding to the eigenvector $\mathbf{V}_{i j n}, \tilde{w}_{i i n}=w_{i i n}$, with the exception of the six 
normal modes representing rotations and translations, for which we set $\tilde{w}_{i i n}=1$. This special norm allows for a better reproduction of the high frequencies related to protons. We found that $k=1$ (introduced in Eq. 17) is optimal to achieve the best fit in reproducing the frequencies with a minor loss of accuracy in determining the energies.

We start optimization of the AVB LHA model for porphyrin by setting $\mathbf{A}_{i i}=\mathbf{T}_{i i}$ for all $i$, and minimize $\Upsilon_{1}^{2}+\Upsilon_{2}^{2}$ with the conjugate gradient method up to the zero value. In this stage, the Hessian parameters A are fixed and not optimized. In the next stage, we optimize all parameters including $\mathrm{A}$, and we minimize the quantity $\Upsilon_{1}^{2}+\Upsilon_{2}^{2}+\Upsilon_{3}^{2} / 20$, until the model reproduces the energies up to $0.11 \mathrm{~kJ} / \mathrm{mol}$, the stationary geometries up to $0.002 \AA$, and the Hessian frequencies up to $16 \mathrm{~cm}^{-1}$. The scaling factor is set to 20 to balance the contribution of these three components. The accuracy of the AVB LHA Hessians before and after optimization is presented in Table 2 . Notice that optimization of Hessians involves only the local minima, and not the saddle points, consistently with the fact, that the off-diagonal elements of the Hamiltonian matrix are parameterized with linear expressions in the LHA model. Nevertheless, the AVB model reproduces qualitatively the DFT Hessians also in the saddle points, see Table 3.

In the first stage of NHA optimization, we adopt and fix the parameters of the LHA model, and optimize the parameters appearing in the expressions (4) and (6) by minimization the root means square (RMS) deviation between AVB-PES and DFT-PES on the two-dimensional protonic map for porphyrin. The optimized NHA parameters are presented in Table S1 of the "Electronic supplementary material" (ESM). In the second stage, we reoptimize the parameters adopted from the LHA model, until the NHA model reproduces the energies up to $0.002 \mathrm{~kJ} / \mathrm{mol}$, the stationary geometries up to $0.0001 \AA$, and the Hessian frequencies up to 1.3 $\mathrm{cm}^{-1}$, see Table 2. We use the same set of parameters from Table S1 of the ESM for all valence states of porphyrin and porphycene, except for the NH distance in non-bonding interaction (6) (1.02 $\AA$ for porphyrin vs. $1.05 \AA$ for porphycene) and the exponential decay parameter in Eq. 8 . The NHA model for porphycene was obtained applying the same schema as porphyrin skipping fitting the parameters appearing in expressions (4) and (6). The accuracy of AVB NHA Hessians before and after optimization is presented in Tables 4 and 5 for the minima and saddle points, respectively. In Fig. 6, we present the protonic maps of the AVB-PES for porphyrin in the LHA and NHA model. The relative RMS deviations between the DFT and AVB protonic maps for both porphyrin and porphycene with energies below 150 $\mathrm{kJ} / \mathrm{mol}$ are in the range of $4-14 \%$ for NHA and $7-35 \%$ for LHA (see the details in Table S2 of the ESM).

In the last step, the quartic angular potential is optimized by fitting the AVB-PES to the DFT energies at geometries from random classical MD snapshots. The applied optimization procedure and in particular the nonharmonic approximation (NHA), in which the protonic generalized coordinates account for the Morse-type potentials of proton-acceptor bonds, allowed for very good reconstruction of the potential energy maps for all representative geometries of the studied systems, including possible translocations of protons (cf. Figs. 4 and 6).

\section{Tests of the optimized AVB2-PES}

In order to examine the parametrized AVB2-PES, the two sets of MD simulations were carried out for the porphycene and porphyrin molecule. We performed the simulations in the Born-Oppenheimer approximation, applying the Verlet algorithm, with the ensuring numerical accuracy time step of $0.1 \mathrm{fs}$, in the NVE ensemble where the total energy corresponds to the room temperature $(\mathrm{T}=298$ $\mathrm{K})$. In order to simulate the power spectra, the dipole moment of the molecule $\mu_{t o t}(t)$ at time $t$ was computed along the trajectories. Then, the total dipolar correlation function was computed, followed by the computation of its Fourier transformation, resulting in the spectrum function depending on frequency (see the details in the ESM). Each simulated spectrum is obtained from 45 of the 20ps classical trajectories. For porphyrin, spectra obtained from our models are in qualitative agreement with the experimental data [1]. It should be noted that experimental peaks around $2305-2450 \mathrm{~cm}^{-1}$ are represented by the AVB2 peak around $2200 \mathrm{~cm}^{-1}$, which does not exist in the Gaussian harmonic spectrum (see in Figs. S2 and S1 of the ESM). If the trans-cis conversion occurs in the dynamics, there should be a small, but detectable peak at $2333 \mathrm{~cm}^{-1}$, that is "the cis fingerprint" [1]. It requires further studies, most likely with much longer MD/AVB2-PES simulations. Also in the case of porphycene, one observes a narrow peak instead of a broad band in the $400-1600 \mathrm{~cm}^{-1}$ region [5], which possibly could appear with much longer MD/AVB2-PES simulations (see in Figs. S3 and S4 of the ESM). Then, we performed the series of MD/AVB2PES simulations with one selected vibrational mode being excited. For porphycene, we applied the excitation along the strongest promoting normal mode $\left(\nu=182 \mathrm{~cm}^{-1}\right)[5,6$, 17]. Starting from the trans state, we performed a series of simulations with the total energy in the range of 40-100 $\mathrm{kJ} / \mathrm{mol}$ with interval $10 \mathrm{~kJ} / \mathrm{mol}$, and in the range of 51-79 $\mathrm{kJ} / \mathrm{mol}$ with an interval of $1 \mathrm{~kJ} / \mathrm{mol}$. We used the Verlet algorithm in the NVE ensemble for 20-ps simulations. The time step of $0.1 \mathrm{fs}$ was used to ensure numerical accuracy. As expected, the distributions of the proton transfers as a function of the initial kinetic energy is monotonically growing. A typical plot of the $\left|c_{i}\right|^{2}$ coefficients representing 
Table 2 Selected vibrational wave numbers (in $\mathrm{cm}^{-1}$ ) of porphyrin computed using the DFT method, implemented in the Gaussian '03 package in the Mt (upper part) and Mc (lower part) geometries, and the deviation between DFT and AVB wave numbers at various stages of parameterization: the LHA and NHA models before and after optimization of Hessians

\begin{tabular}{|c|c|c|c|c|c|}
\hline Mode & $v$ & $\Delta v_{\text {before }}^{L H A}$ & $\Delta v_{\text {before }}^{N H A}$ & $\Delta v_{\text {after }}^{L H A}$ & $\Delta v_{\text {after }}^{N H A}$ \\
\hline 10 & 99.0 & 14.7 & -39.6 & 16.3 & -0.2 \\
\hline 11 & 124.7 & 2.1 & 6.3 & -0.2 & -0.0 \\
\hline 17 & 292.4 & 19.9 & -27.7 & 0.8 & -0.0 \\
\hline 20 & 316.6 & 5.1 & 0.3 & 0.5 & -0.0 \\
\hline 24 & 396.0 & 8.0 & -6.0 & 0.2 & 0.0 \\
\hline 29 & 631.0 & 0.8 & 13.2 & -0.0 & 0.0 \\
\hline 32 & 680.6 & -0.0 & 6.1 & -0.0 & 0.0 \\
\hline 41 & 748.5 & 0.9 & 8.2 & 0.0 & -0.0 \\
\hline 44 & 791.8 & 1.8 & -12.6 & 0.0 & -0.0 \\
\hline 48 & 797.0 & 2.1 & -8.3 & 0.0 & -0.0 \\
\hline 49 & 808.4 & 0.2 & 7.9 & -0.0 & -0.0 \\
\hline 59 & 972.7 & 0.8 & -13.7 & -0.1 & 0.0 \\
\hline 60 & 976.3 & 6.1 & 0.3 & -0.0 & -0.0 \\
\hline 62 & 999.2 & -2.9 & -30.5 & -0.1 & -0.0 \\
\hline 63 & 1011.8 & 3.6 & -30.2 & 0.2 & 0.0 \\
\hline 71 & 1169.7 & -0.3 & -8.9 & -0.1 & -0.0 \\
\hline 73 & 1188.6 & -2.4 & -17.9 & -0.1 & 0.0 \\
\hline 77 & 1261.9 & -13.8 & -34.5 & -0.4 & -0.0 \\
\hline 78 & 1269.0 & -9.4 & -48.2 & -0.2 & -0.0 \\
\hline 92 & 1539.1 & -4.6 & -7.5 & 0.0 & -0.0 \\
\hline 98 & 1640.6 & -5.4 & -3.6 & -0.1 & -0.0 \\
\hline 99 & 1645.3 & -6.7 & -4.8 & 0.0 & -0.0 \\
\hline 113 & 3557.3 & -189.9 & -13.8 & -2.3 & 0.0 \\
\hline 114 & 3600.3 & -151.5 & -5.6 & -1.5 & -0.0 \\
\hline 9 & 93.6 & 0.3 & 30.6 & 0.1 & -0.0 \\
\hline 10 & 112.6 & 2.7 & 32.1 & 0.0 & -0.0 \\
\hline 11 & 139.2 & 7.9 & -49.1 & 0.3 & 0.0 \\
\hline 12 & 148.6 & 3.3 & 16.4 & 0.6 & -0.0 \\
\hline 16 & 203.8 & 2.4 & 22.3 & 0.2 & -0.0 \\
\hline 19 & 315.2 & 7.0 & -33.0 & -0.6 & 0.0 \\
\hline 29 & 623.4 & 2.5 & 33.4 & -0.0 & 0.0 \\
\hline 34 & 699.1 & -1.3 & 16.5 & -0.0 & 0.0 \\
\hline 37 & 721.0 & 1.5 & -43.7 & -0.2 & 0.0 \\
\hline 38 & 722.4 & -2.4 & 64.2 & 0.0 & -0.0 \\
\hline 47 & 799.3 & -0.3 & 347.7 & 0.0 & 0.0 \\
\hline 50 & 835.0 & -5.3 & 395.4 & -0.0 & 0.0 \\
\hline 59 & 969.7 & -3.3 & -49.8 & -0.1 & 0.0 \\
\hline 60 & 976.1 & 11.1 & 18.4 & -0.0 & 0.0 \\
\hline 62 & 985.5 & 1.2 & -94.9 & -0.3 & 0.0 \\
\hline 72 & 1177.9 & 0.7 & -29.5 & 0.0 & 0.0 \\
\hline 77 & 1268.8 & -1.8 & -22.0 & -0.1 & -0.0 \\
\hline 78 & 1293.9 & -11.6 & -32.1 & -0.5 & 0.0 \\
\hline 106 & 3230.4 & 0.3 & -24.7 & -0.0 & -0.0 \\
\hline 109 & 3251.5 & 0.1 & -72.8 & -0.0 & -0.0 \\
\hline 110 & 3251.8 & -68.2 & -20.7 & -0.0 & -0.0 \\
\hline 113 & 3313.6 & -185.9 & -49.5 & -2.1 & 0.1 \\
\hline 114 & 3346.3 & -81.3 & -81.4 & -1.1 & 0.0 \\
\hline
\end{tabular}

Only those modes which have at least one deviation greater than $10 \mathrm{~cm}^{-1}$ are presented 
Table 3 Selected vibrational wave numbers (in $\mathrm{cm}^{-1}$ ) of porphyrin computed using the DFT method, implemented in the Gaussian '03 package in the S1 (upper part) and S2 (lower part) geometries, and the deviation between DFT and AVB wave numbers (see Table 2)

\begin{tabular}{|c|c|c|c|c|c|}
\hline Mode & $v$ & $\Delta v_{\text {before }}^{L H A}$ & $\Delta v_{\text {before }}^{N H A}$ & $\Delta v_{\text {after }}^{L H A}$ & $\Delta v_{\text {after }}^{N H A}$ \\
\hline 9 & 95.8 & -1.1 & 36.1 & -0.9 & 26.9 \\
\hline 10 & 127.2 & -4.1 & 24.7 & -7.0 & 25.2 \\
\hline 13 & 178.0 & 4.6 & 0.6 & 24.1 & -0.6 \\
\hline 14 & 184.9 & 6.6 & 25.7 & 6.0 & 17.5 \\
\hline 16 & 222.8 & -6.4 & 28.5 & -7.1 & 24.4 \\
\hline 23 & 407.3 & -28.5 & -37.7 & -29.0 & -34.6 \\
\hline 29 & 658.4 & -25.6 & 4.6 & -26.4 & 0.5 \\
\hline 37 & 731.6 & 1.5 & -22.0 & 1.0 & -5.7 \\
\hline 40 & 756.5 & -41.5 & 18.7 & -40.8 & 16.2 \\
\hline 42 & 778.9 & -23.7 & 8.3 & -23.2 & 7.0 \\
\hline 48 & 813.9 & -15.9 & 304.5 & -15.9 & 47.5 \\
\hline 52 & 860.0 & 2.0 & 2.0 & 1.9 & 48.8 \\
\hline 58 & 977.7 & -2.5 & -67.8 & -1.7 & -18.4 \\
\hline 64 & 1037.7 & 30.7 & 7.9 & 26.9 & 10.9 \\
\hline 72 & 1181.9 & -272.7 & 408.7 & -365.8 & 459.3 \\
\hline 76 & 1253.3 & 3.7 & -25.5 & 4.2 & 3.2 \\
\hline 78 & 1305.1 & -19.5 & -41.1 & -9.2 & -16.2 \\
\hline 89 & -1479.8 & -166.5 & -420.9 & -287.2 & -119.9 \\
\hline 101 & 1869.9 & 616.8 & -34.8 & 675.0 & 177.9 \\
\hline 114 & 3333.7 & -64.2 & -66.2 & 50.0 & 2.0 \\
\hline 9 & 103.4 & -8.5 & 48.3 & -8.2 & 46.6 \\
\hline 11 & 156.4 & 16.6 & 31.9 & 13.6 & 32.5 \\
\hline 13 & 193.0 & 12.7 & 48.8 & 11.9 & 42.6 \\
\hline 15 & 208.9 & 2.8 & -36.1 & 12.5 & -13.3 \\
\hline 16 & 222.5 & -1.9 & 39.7 & -1.9 & 33.9 \\
\hline 20 & 353.6 & 39.8 & 11.4 & 36.1 & 11.5 \\
\hline 21 & 375.5 & -31.6 & -56.1 & -31.8 & -52.6 \\
\hline 22 & 380.2 & -16.8 & -42.2 & -15.9 & -33.4 \\
\hline 26 & 438.8 & -41.4 & -67.2 & -39.2 & -62.5 \\
\hline 30 & 676.8 & -40.4 & 1.1 & -40.2 & 0.1 \\
\hline 36 & 714.2 & -31.3 & 0.1 & -30.8 & 0.5 \\
\hline 42 & 784.2 & -71.0 & 3.3 & -70.5 & 2.7 \\
\hline 49 & 843.2 & -33.3 & 7.3 & 7.4 & 7.3 \\
\hline 59 & 1013.0 & -21.9 & -31.6 & -21.0 & -16.4 \\
\hline 61 & 1036.0 & 40.4 & 5.2 & 33.5 & 5.9 \\
\hline 62 & 1038.0 & 34.2 & 16.0 & 29.9 & 15.1 \\
\hline 69 & 1161.0 & -371.1 & 503.0 & -370.9 & 507.4 \\
\hline 72 & 1209.9 & -337.7 & 485.1 & -399.1 & 491.7 \\
\hline 75 & 1233.9 & -34.3 & -39.2 & -33.1 & -34.7 \\
\hline 81 & -1374.0 & -125.9 & -88.3 & -233.2 & -115.4 \\
\hline 87 & -1466.9 & -252.3 & -390.6 & -385.6 & -70.8 \\
\hline 89 & 1476.4 & -26.1 & -32.0 & -24.7 & -26.8 \\
\hline 101 & 1857.5 & 642.9 & 36.1 & 702.1 & 234.3 \\
\hline 102 & 1893.0 & 614.1 & -49.3 & 693.2 & 205.3 \\
\hline
\end{tabular}

Only those modes which have at least one deviation greater than $20 \mathrm{~cm}^{-1}$ for $\mathrm{S} 1$ and $30 \mathrm{~cm}^{-1}$ for $\mathrm{S} 2$ are presented 
Table 4 Selected vibrational wave numbers (in $\mathrm{cm}^{-1}$ ) of porphycene computed using the DFT method, implemented in the Gaussian '03 package in the Mt (upper part) and Mc (lower part) geometries, and the deviation between DFT and AVB wave numbers at various stages of parameterization: LHA and NHA model before and after optimization of Hessians

\begin{tabular}{|c|c|c|c|c|c|}
\hline Mode & $v$ & $\Delta v_{\text {before }}^{L H A}$ & $\Delta v_{\text {before }}^{N H A}$ & $\Delta v_{\text {after }}^{L H A}$ & $\Delta v_{\text {after }}^{N H A}$ \\
\hline 9 & 88.5 & 0.0 & 14.7 & 0.0 & 0.0 \\
\hline 10 & 119.4 & 0.0 & 5.3 & -0.0 & -0.0 \\
\hline 11 & 136.8 & 0.3 & 8.6 & 0.0 & 0.0 \\
\hline 13 & 186.7 & 0.2 & 22.1 & 1.8 & 0.5 \\
\hline 46 & 832.8 & -6.2 & -5.8 & -0.2 & -0.0 \\
\hline 49 & 877.2 & -7.9 & -5.5 & -0.2 & -0.0 \\
\hline 55 & 933.4 & -0.0 & 24.4 & 0.0 & 0.0 \\
\hline 56 & 947.4 & -11.7 & -7.0 & -0.0 & -0.0 \\
\hline 58 & 957.9 & -0.0 & 93.0 & 0.0 & 0.0 \\
\hline 62 & 999.3 & -0.0 & 111.6 & -0.0 & 0.0 \\
\hline 63 & 1016.4 & -5.9 & -0.9 & -0.1 & -0.0 \\
\hline 92 & 1531.3 & -0.2 & 8.2 & 0.0 & 0.0 \\
\hline 99 & 1631.3 & -0.7 & 7.0 & 0.0 & -0.0 \\
\hline 101 & 2894.8 & -497.9 & -65.4 & -2.4 & -0.1 \\
\hline 102 & 2895.1 & -497.4 & -41.6 & -1.3 & -0.0 \\
\hline 12 & 143.5 & 23.3 & 1.0 & 0.1 & 0.0 \\
\hline 15 & 205.5 & -7.0 & 23.9 & 2.8 & 0.9 \\
\hline 17 & 244.2 & 12.5 & 1.4 & 0.1 & 0.0 \\
\hline 55 & 934.3 & -18.2 & -11.6 & -0.2 & -0.1 \\
\hline 59 & 977.9 & -21.4 & 36.3 & -0.0 & 0.0 \\
\hline 64 & 1052.0 & -33.1 & 34.1 & -0.0 & 0.0 \\
\hline 69 & 1117.5 & 12.4 & -0.2 & -0.0 & 0.0 \\
\hline 77 & 1301.4 & 11.7 & -2.0 & 0.0 & 0.0 \\
\hline 78 & 1311.9 & -11.2 & -0.7 & 0.0 & -0.0 \\
\hline 80 & 1334.0 & 10.8 & 0.6 & -0.1 & 0.0 \\
\hline 83 & 1400.4 & -15.1 & -0.4 & 0.0 & -0.0 \\
\hline 93 & 1537.6 & 10.5 & 1.7 & -0.1 & -0.0 \\
\hline 94 & 1561.0 & -11.2 & 0.6 & 0.1 & -0.0 \\
\hline 95 & 1575.0 & -10.1 & 0.5 & -0.1 & -0.0 \\
\hline 98 & 1628.2 & -16.3 & 1.5 & 0.0 & -0.0 \\
\hline 99 & 1628.4 & -13.5 & -0.5 & -0.1 & -0.0 \\
\hline 100 & 1673.4 & -26.9 & 0.7 & -0.2 & -0.0 \\
\hline 101 & 2596.1 & -401.5 & -22.2 & -0.3 & -0.1 \\
\hline 102 & 2634.2 & -513.0 & -44.2 & -1.4 & -0.2 \\
\hline
\end{tabular}

Only those modes which have at least one deviation greater than $5 \mathrm{~cm}^{-1}$ for $\mathrm{Mt}$ (and $10 \mathrm{~cm}^{-1}$ for Mc ) are presented

an occupation measure of the trans minima (blue and magenta) and the cis minima (green) for porphycene is presented in Fig. 7. We can observe many proton-transfer events in the first $5 \mathrm{ps}$, when the most excitation energy is set into the promoting normal mode, and after this time other modes gain energy of the initially excited one and the proton-transfer event is not so frequent, as already noted by Walewski [17]. 
Table 5 Selected vibrational wave numbers (in $\mathrm{cm}^{-1}$ ) of porphycene computed using the DFT method, implemented in the Gaussian '03 package in the S1 (upper part) and S2 (lower part) geometries, and the deviation betwxeen DFT and AVB wave numbers (see Table 4)

\begin{tabular}{|c|c|c|c|c|c|}
\hline Mode & $v$ & $\Delta v_{\text {before }}^{L H A}$ & $\Delta v_{\text {before }}^{N H A}$ & $\Delta v_{\text {after }}^{L H A}$ & $\Delta v_{\text {after }}^{N H A}$ \\
\hline 9 & 89.4 & -0.8 & 26.8 & -0.8 & 32.1 \\
\hline 10 & 120.9 & -1.2 & 20.1 & -2.3 & 20.7 \\
\hline 11 & 146.3 & 1.7 & 15.2 & 2.6 & 15.9 \\
\hline 12 & 151.8 & 12.4 & 13.3 & 15.1 & 14.3 \\
\hline 14 & 208.7 & -0.0 & 15.5 & -1.4 & 16.4 \\
\hline 15 & 214.1 & -1.6 & 15.0 & -2.0 & 15.3 \\
\hline 16 & 240.7 & -15.8 & 9.2 & 11.1 & 10.0 \\
\hline 17 & 250.1 & 3.2 & 8.2 & 86.3 & 2.7 \\
\hline 20 & 340.9 & -3.9 & -6.6 & 16.6 & -6.8 \\
\hline 21 & 365.4 & -14.0 & -3.4 & 4.3 & -2.4 \\
\hline 22 & 370.9 & -0.0 & 1.4 & 35.7 & 0.9 \\
\hline 24 & 401.6 & 5.9 & 4.2 & 65.5 & 4.5 \\
\hline 25 & 492.5 & -1.0 & -11.0 & 6.1 & -11.1 \\
\hline 27 & 495.4 & 3.2 & 5.9 & 12.1 & 6.2 \\
\hline 60 & 1020.3 & -2.3 & -11.5 & 5.2 & -10.5 \\
\hline 61 & 1020.5 & -83.2 & 158.4 & -62.0 & 176.2 \\
\hline 62 & 1032.5 & -7.5 & -5.2 & 10.7 & -4.5 \\
\hline 70 & -1188.3 & -117.6 & -17.6 & -116.6 & -55.4 \\
\hline 75 & 1236.3 & -231.2 & 399.6 & -206.6 & 432.0 \\
\hline 81 & 1343.8 & 17.1 & -0.0 & 16.6 & 0.9 \\
\hline 100 & 1658.1 & -20.1 & 7.7 & -9.7 & 8.4 \\
\hline 101 & 1810.1 & -50.1 & 202.1 & 97.4 & 251.3 \\
\hline 102 & 2595.2 & -383.2 & -41.4 & -117.7 & -15.4 \\
\hline 9 & 91.6 & -2.9 & 51.3 & -2.9 & 55.9 \\
\hline 10 & 121.8 & -1.8 & 19.1 & -2.9 & 20.2 \\
\hline 11 & 153.7 & 0.9 & 21.0 & 0.6 & 20.2 \\
\hline 12 & 156.6 & 18.2 & 24.4 & 25.2 & 25.2 \\
\hline 14 & 209.4 & -1.2 & 24.7 & -1.7 & 27.5 \\
\hline 15 & 215.0 & 1.5 & 22.1 & -0.0 & 21.0 \\
\hline 17 & 282.5 & -28.6 & -4.2 & 76.4 & -18.0 \\
\hline 20 & 359.8 & -11.9 & -16.0 & -8.7 & -15.1 \\
\hline 21 & 369.4 & -17.0 & -0.5 & 1.1 & 0.5 \\
\hline 22 & 377.7 & -6.1 & -4.5 & 189.5 & -5.4 \\
\hline 27 & 506.4 & -1.8 & -17.3 & 2.2 & -16.4 \\
\hline 61 & 1050.6 & -19.8 & -30.5 & 6.6 & -29.9 \\
\hline 63 & -1064.1 & -76.6 & -13.1 & 46.8 & -24.5 \\
\hline 71 & -1202.4 & -191.9 & -24.8 & 21.5 & -45.0 \\
\hline 72 & 1204.1 & -270.2 & 423.6 & -249.8 & 506.3 \\
\hline 76 & 1253.0 & -251.1 & 403.9 & -227.3 & 485.0 \\
\hline 79 & 1321.8 & 5.3 & 1.6 & 15.1 & 2.7 \\
\hline 82 & 1369.8 & -10.7 & -19.4 & 8.6 & -18.2 \\
\hline 101 & 1792.1 & 39.9 & 205.8 & 107.0 & 302.2 \\
\hline 102 & 1832.2 & -119.2 & 226.9 & 80.1 & 315.0 \\
\hline
\end{tabular}

Only those modes which have at least one deviation greater than $10 \mathrm{~cm}^{-1}$ for $\mathrm{S} 1$ and $15 \mathrm{~cm}^{-1}$ for $\mathrm{S} 2$ are presented 


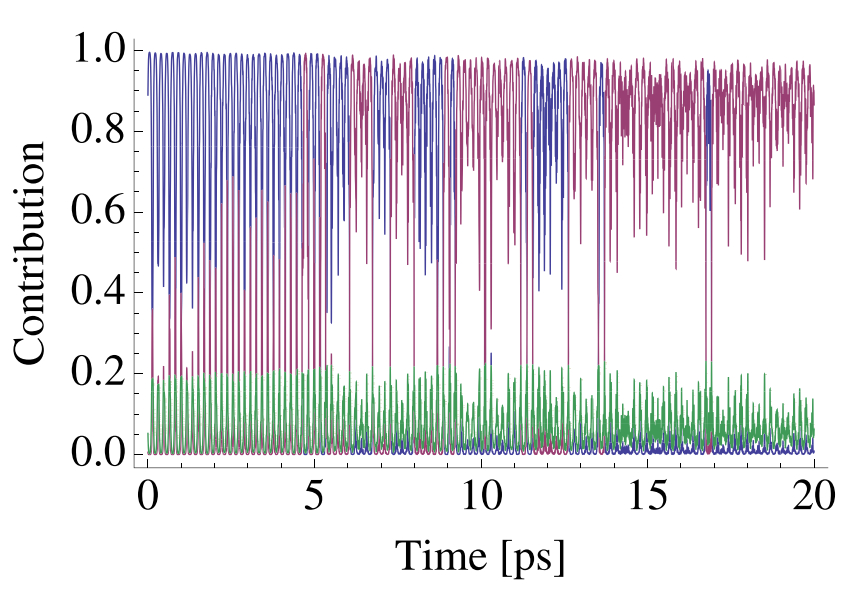

Fig. 7 A typical plot of the $\left|c_{i}\right|^{2}$ coefficients representing occupation probabilities of the trans minima (blue and magenta) and of the cis minima (green) for porphycene from the MD/AVB2-PES simulations

\section{Conclusions}

This study presents a novel theoretical model (AVB2) a very fast quantum generator of the molecular potential energy based on the approximate valence bond method. AVB2 was parameterized using results of quantum mechanical computations at the DFT-B3LYP/6-31G (d, p) level. It should be emphasized that for the purposes of parameterization of AVB2 any other DFT or conventional ab initio method, which computes the potential energy at the BornOppenheimer method (Q-PES), Q-PES gradients and QPES Hessian elements, as well as effective atomic charges for local energy minima and transition states, can be used. Two difficult molecular systems were selected for the validation of the method: porphyrin and porphycene molecules. In their molecular cavities, double proton transfer processes occur, which are strongly correlated with changes of the electronic charge density. In the parameterization, the low-energy tautomeric structures of the above-mentioned systems were used. In contrast to many other methods which propose direct parameterization of Q-PESes, in the case of AVB2 we propose parameterization of the low-dimensional electronic Hamiltonian matrix elements. The AVB2 method, and in particular its nonharmonic version (NHA), very well reproduce the potential energy maps for all representative geometries of the studied systems, including possible proton translocations (compare Figs. 4 and 6). An additional, initial validation of the method was also performed by comparing the experimental and theoretical infrared spectra of the molecules. Qualitative agreement of spectra was obtained. It should be emphasized, however, that quantitative compliance is not yet possible, mainly for two reasons. First, conventional, classical formalism of the total dipolar correlation function and its Fourier transformation has known theoretical limitations, and in addition it would also be required to carry out much longer MD/AVB simulations to better reproduce low-frequency transitions. Secondly, it should be noted that motions of the protons are largely quantum in nature. Therefore, infrared spectra simulations have to go beyond the classical models and use quantum molecular dynamics simulations, i.e., the implementation of a QD/AVB2-PES model. This topic of research, as well as the possibility of extending the AVB2 parameterization to electronic excited states, are the subject of our further research.

Acknowledgments This study was supported by IMDiK PAN (Z526, topic 21) as well as BST-176600/BF/22 (UW) funds. The computations were carried out using the infrastructure of ICM, University of Warsaw.

Open Access This article is distributed under the terms of the Creative Commons Attribution 4.0 International License (http:// creativecommons.org/licenses/by/4.0/), which permits unrestricted use, distribution, and reproduction in any medium, provided you give appropriate credit to the original author(s) and the source, provide a link to the Creative Commons license, and indicate if changes were made.

\section{References}

1. Baker J, Kozlowski P, Jarzecki A, Pulay P (1997) The innerhydrogen migration in free base porphyrin. Theor Chem Acc: Theory Comput Model 97:59-66

2. Bala P, Grochowski P, Nowinski K, Lesyng B, McCammon JA (2000) Quantum-dynamical picture of a multi-step enzymatic process reaction catalyzed by phospholipase a2. Biophys $\mathbf{J}$ 79:1253-1262

3. Becke AD (1993) Density-functional thermochemistry. III. The role of exact exchange. J Chem Phys 98:5648-5652

4. Frisch MJ, Trucks GW, Schlegel HB, Scuseria GE, Robb MA, Cheeseman JR, Montgomery JA Jr, Vreven T, Kudin KN, Burant JC, Millam JM, Iyengar SS, Tomasi J, Barone V, Mennucci B, Cossi M, Scalmani G, Rega N, Petersson GA, Nakatsuji H, Hada M, Ehara M, Toyota K, Fukuda R, Hasegawa J, Ishida M, Nakajima T, Honda Y, Kitao O, Nakai H, Klene M, Li X, Knox JE, Hratchian HP, Cross JB, Bakken V, Adamo C, Jaramillo J, Gomperts R, Stratmann RE, Yazyev O, Austin AJ, Cammi R, Pomelli C, Ochterski JW, Ayala PY, Morokuma K, Voth GA, Salvador P, Dannenberg JJ, Zakrzewski VG, Dapprich $S$, Daniels AD, Strain MC, Farkas O, Malick DK, Rabuck AD, Raghavachari K, Foresman JB, Ortiz JV, Cui Q, Baboul AG, Clifford S, Cioslowski J, Stefanov BB, Liu G, Liashenko A, Piskorz P, Komaromi I, Martin RL, Fox DJ, Keith T, Al-Laham MA, Peng CY, Nanayakkara A, Challacombe M, Gill PMW, Johnson B, Chen W, Wong MW, Gonzalez C, Pople JA (2004) Gaussian 03, Revision C.02. Gaussian, Inc., Wallingford

5. Gawinkowski S, Walewski L, Vdovin A, Slenczka A, Rols S, Johnson MR, Lesyng B, Waluk J (2012) Vibrations and hydrogen bonding in porphycene. Phys Chem Chem Phys 14:5489-5503

6. Gil M, Waluk J (2007) Vibrational gating of double hydrogen tunneling in porphycene. J Am Chem Soc 129:1335-1341

7. Grochowski P (2008) Rotational symmetry of the molecular potential energy in the Cartesian coordinates. Theor Chem Acc: Theory Comput Model 121:257-266

8. Grochowski P, Lesyng B (2003) Extended Hellmann-Feynman forces, canonical representations, and exponential propagators in 
the mixed quantum-classical molecular dynamics. J Chem Phys 119:11541-11555

9. Grochowski P, Lesyng B, Bala P, McCammon J (1996) Density functional based parametrization of a valence bond method and its applications in quantum-classical molecular dynamics simulations of enzymatic reactions. Int J Quantum Chem 60:11431164

10. Hollebeek T, Ho TS, Rabitz H (1999) Constructing multidimensional molecular potential energy surfaces from ab initio data. Annu Rev Phys Chem 50:537-570

11. Hutter J (2015) Copyright IBM Corp 1990-2015, Copyright MPI für Festkörperforschung Stuttgart 1997-2001. http://www.cpmd. org/

12. Ischtwan J, Collins MA (1994) Molecular potential energy surfaces by interpolation. J Chem Phys 100:8080-8088

13. Lee F, Warshel A (1992) A local reaction field method for fast evaluation of long-range electrostatic interactions in molecular simulations. J Chem Phys 97:3100-3107

14. Thompson KC, Jordan MJT, Collins MA (1998a) Molecular potential energy surfaces by interpolation in cartesian coordinates. J Chem Phys 108(2):564-578

15. Thompson KC, Jordan MJT, Collins MA (1998b) Polyatomic molecular potential energy surfaces by interpolation in local internal coordinates. J Chem Phys 108(20):8302-8316
16. Trylska J, Grochowski P, Geller M (2001) Parameterization of the approximate valence bond (AVB) method to describe potential energy surface in the reaction catalyzed by HIV-1 protease. Int $\mathbf{J}$ Quantum Chem 82:86-103

17. Walewski L, Waluk J, Lesyng B (2010) Car-Parrinello molecular dynamics study of the intramolecular vibrational mode-sensitive double proton-transfer mechanisms in porphycene. J Phys Chem A 114(6):2313-2318

18. Warshel A (1991) Computer modeling of chemical reactions in enzymes and solutions. Wiley, New York

19. Warshel A, Weiss RM (1980) Vibrations and hydrogen bonding in porphycene. J Am Chem Soc 102:6218-6226

20. Yoshikawa T, Sugawara S, Takayanagi T, Shiga M, Tachikawa M (2010) Theoretical study on the mechanism of double proton transfer in porphycene by path-integral molecular dynamics simulations. Chem Phys Lett 496:14-19

21. Yoshikawa T, Sugawara S, Takayanagi T, Shiga M, Tachikawa M (2012) Quantum tautomerization in porphycene and its isotopomers: Path-integral molecular dynamics simulations. Chem Phys Lett 394:46-51

Publisher's note Springer Nature remains neutral with regard to jurisdictional claims in published maps and institutional affiliations. 